\title{
Le cas français : approfondir les connaissances empiriques pour mieux cibler la formation
}

To study thoroughly empirical knowledges to improve continuing training in

France

\section{Bruno Crépon et Marc Ferracci}

\section{OpenEdition \\ Journals}

Édition électronique

URL : http://journals.openedition.org/travailemploi/3607

DOI : 10.4000/travailemploi.3607

ISSN : $1775-416 X$

Éditeur

DARES - Ministère du Travail

Édition imprimée

Date de publication : 5 juin 2009

Pagination : 107-111

ISSN : 0224-4365

Référence électronique

Bruno Crépon et Marc Ferracci, « Le cas français : approfondir les connaissances empiriques pour mieux cibler la formation », Travail et Emploi [En ligne], 118 | avril-juin 2009, mis en ligne le 03 décembre 2010, consulté le 30 avril 2019. URL : http://journals.openedition.org/travailemploi/3607 ; DOI : 10.4000/travailemploi.3607 


\title{
Le cas français: approfondir les connaissances empiriques pour mieux cibler la formation
}

\author{
Bruno Crépon (*), Marc Ferracci (**)
}

En matière de formation professionnelle, l'OCDE défend depuis quelques années une stratégie volontariste. Sur la base d'une observation précise des politiques menées dans les pays membres, et en mobilisant les résultats issus de la littérature économique, les différentes Perspectives de l'emploi ayant été publiées depuis le début des années 2000 (1) établissent un double constat:

-il existe de fortes inégalités entre individus concernant l'accès aux programmes de formation. Ces inégalités touchent aussi bien la population des salariés que celle des chômeurs;

-l'effet de la formation sur les perspectives d'emploi et de revenus des individus qui en bénéficient est généralement positif.

De ce constat découle un objectif global: accroître l'accès à la formation, pour les salariés comme pour les demandeurs d'emploi, et améliorer l'efficacité moyenne des mesures à ressources données.

Cette contribution fournit dans un premier temps une appréciation sur le diagnostic qui fonde la stratégie de l'OCDE. Elle cherche dans un second temps à préciser les contours de cette stratégie dans le cas français, en insistant sur la nécessité d'évaluer plus systématiquement les mesures de formation, et de cibler ces dernières sur les publics les plus susceptibles d'en tirer profit. Il apparaît en effet que la France est en retard sur la plupart des pays industrialisés en matière d'évaluation de la formation. Des évaluations plus systématiques permettraient d'améliorer l'efficacité des dispositifs existants, et sans doute d'accroître sensiblement le rendement de la dépense de formation.

\footnotetext{
* CREST, Centre de recherches en économie et en statistique; crepon@ensae.fr

** CREST, Centre de recherches en économie et en statistique ; ferracci@ensae.fr

(1) Voir en particulier les éditions 2003 (chapitre 5) et 2006 (chapitre 3) pour la formation des salariés, et l'édition 2005 (chapitre 4) pour les programmes de formation destinés aux chômeurs.
}

\section{Le diagnostic de l'OCDE}

L'existence d'inégalités d'accès à la formation professionnelle dans les pays de l'OCDE est bien documentée. Cet accès est positivement corrélé avec le niveau d'éducation initiale. En France, les salariés sans diplôme ont ainsi un taux de participation à la formation plus de trois fois inférieur à celui des salariés titulaires d'un diplôme de l'enseignement supérieur. Pour les demandeurs d'emploi, la situation est similaire, quoique moins défavorable aux sans diplôme: leur taux de participation est deux fois inférieur à celui des diplômés du supérieur(2). En outre, il est commun d'observer que la participation décroît avec l'âge, et que les chômeurs ont par ailleurs moins accès à la formation que les salariés en emploi.

\section{Des inégalités incontestables, qui tiennent en partie au fonctionnement des dispositifs de formation}

Dans le système français, ces inégalités résultent, pour partie au moins, des règles qui régissent le dispositif institutionnel de formation. S'agissant de la formation des salariés, le système français est caractérisé, depuis la loi du 16 juillet 1971, par l'obligation faite aux entreprises de dédier une fraction de leur masse salariale aux actions de formation. Cette obligation de dépenser de manière indifférenciée mène les entreprises à diriger les actions de formation vers les individus les plus qualifiés, qui sont aussi ceux pour lesquels les rendements de la formation sont les plus élevés. De ce point de vue, le système français apparaît profondément antiredistributif.

S'agissant de la formation des chômeurs, le système français se caractérise par une complexité institutionnelle certaine. Les actions de formation professionnelle sont en effet proposées et financées par trois acteurs distincts, dont la coordination est

(2) Source: enquête «Formation continue», 2000. 
imparfaite: l'État, les conseils régionaux, et l'assurance chômage. La conséquence en est que l'offre de formation est globalement peu lisible, ce qui est en soi un facteur de discrimination des individus les moins diplômés, ou les moins motivés(3). Les inégalités d'accès liées à l'éducation initiale s'en trouvent accrues.

Le constat de l'OCDE quant aux inégalités d'accès à la formation apparaît donc peu contestable, et se trouve confirmé par le fonctionnement du système français de formation.

\section{Des conclusions sans doute optimistes quant à l'impact des mesures de formation}

L'OCDE semble tenir pour acquis le fait que la formation professionnelle génère des rendements positifs, à la fois pour les individus bénéficiaires, pour les firmes, et pour l'ensemble de la société (voir notamment OCDE, 2006, pp. 114-115). Une analyse détaillée de la littérature empirique ne permet pourtant pas de tirer des conclusions définitives à ce sujet.

\section{La formation en entreprise: \\ la difficulté à mettre en évidence un lien causal entre formation, salaires et productivité}

L'identification d'un effet spécifique de la formation des salariés sur la productivité et les salaires de leurs bénéficiaires se heurte à de lourds problèmes méthodologiques, qui n'ont été qu'imparfaitement résolus jusqu'à présent. En effet, la mise en évidence d'une corrélation entre des périodes de formation professionnelle et des hausses de salaires ne peut en aucun cas s'interpréter comme une relation causale entre formation professionnelle et salaire.

Les études qui mettent en œuvre une stratégie d'identification valide trouvent généralement que la formation a des effets faibles, voire nuls sur les salaires de ses bénéficiaires. Par exemple, Goux et MAURIN (2000) trouvent qu'en France, le supplément de salaire des employés bénéficiant de la formation professionnelle provient uniquement de leurs caractéristiques personnelles. En d'autres termes, ce sont les salariés les plus productifs qui suivent les stages de formation et qui obtiennent les plus fortes hausses de salaire. En revanche, Goux et MAURIN (2000) montrent que sur les mêmes données la méthode des moindres carrés ordinaires prédit qu'une semaine de formation continue augmente le salaire du bénéficiaire de $5 \%$. Un tel chiffre apparaît très nettement surévalué si l'on considère qu'une année entière d'éducation accroît le salaire dans une fourchette comprise entre $5 \%$ et $15 \%$.

(3) Voir à ce sujet l'étude récente consacrée par la DARES (2006) au processus de participation des chômeurs aux programmes de formation.
Ces résultats rejoignent ceux mis en évidence par LeUven (2004), qui souligne la difficulté d'obtenir une source de variation exogène dans la participation à la formation permettant d'identifier un effet spécifique de cette dernière. LeUven (2004) souligne en outre le fait que les évaluations existantes estiment généralement des rendements salariaux moyens de la formation alors que ces rendements sont hétérogènes par nature. De ce point de vue, les travaux reposant sur des modèles à effets fixes gagneraient à être complétés par des évaluations par appariement, lesquelles permettent de tenir compte de l'hétérogénéité des rendements de la formation.

Il convient par conséquent d'interpréter avec prudence les résultats d'études qui ne contrôlent pas la sélectivité issue de facteurs observables, mais aussi de facteurs inobservables par l'économètre. De ce point de vue, les travaux de l'OCDE gagneraient à distinguer de manière plus claire les études selon la robustesse de leurs résultats (4).

Dans le cadre français, les perspectives de l'évaluation sont toutefois limitées par le manque de données longitudinales portant sur des périodes suffisamment longues, alors que les méthodes permettant d'exploiter de telles données existent(5). Sur ce point, il n'est pas inutile de rappeler que des sommes considérables sont investies chaque année dans le système de formation continue des adultes (6). Une part modeste de ces ressources gagnerait à financer des travaux d'évaluation permettant d'améliorer le rendement global de la dépense.

\section{La formation des chômeurs: de la nécessité de tenir compte de l'hétérogénéité des effets dans le temps, et dans la population}

Il est primordial de distinguer les effets de la formation des demandeurs d'emploi dans le temps. À court terme, la formation des chômeurs peut affecter le taux d'emploi de ses bénéficiaires en modifiant la durée de leur épisode de chômage. Les études ayant évalué l'impact de la formation des demandeurs d'emploi sur la durée du chômage se sont multipliées ces dernières années. Les résultats en sont globalement décevants, même s'ils apparaissent hétérogènes suivant les publics. Pour les interpréter, il est nécessaire de rappeler que les programmes de formation à destination des chômeurs peuvent affecter leur

(4) À titre d'exemple, à la page 114 des Perspectives de l'emploi 2006, une référence à la revue de littérature de LeUven (2004) vient appuyer l'idée que les résultats empiriques convergeraient pour montrer des effets positifs de la formation sur la productivité et les salaires. Cette revue de littérature insiste pourtant sur la fragilité des résultats existants, et sur la difficulté à mettre en évidence un effet proprement causal de la formation.

(5) Voir en particulier BrodATY, CRÉPON et Fougère (2005) pour un panorama complet des méthodes d'évaluation des politiques d'emploi.

(6) Les dépenses de formation professionnelle pour les salariés s'élevaient en 2004 à près de 20 milliards d'euros. 
taux de transition vers l'emploi de façon différente au cours du temps. Les effets en jeu sont potentiellement contradictoires, ce qui rend l'impact global de la formation a priori ambigu.

Avant qu'elle ne débute, la formation peut avoir pour effet d'accroître l'intensité de la recherche d'emploi, dès lors que le fait d'être formé est assimilé à une perte de loisir, et donc d'utilité. De ce point de vue, la formation agirait comme une «menace», qui inciterait les chômeurs à retrouver un emploi plus vite. Cet effet de menace a été mis en évidence dans des études récentes (7), et assimilé dans les travaux de l'OCDE à un effet de «motivation» (OCDE, 2005), susceptible de justifier l'extension des programmes à une population plus large.

Pendant qu'elle se déroule, la formation des chômeurs exerce en revanche un effet de rétention (lock-in), qui voit les individus formés réduire l'intensité de leur recherche d'emploi. Cet effet de rétention est observé de manière systématique dans les évaluations empiriques.

Après qu'elle a pris fin, la formation peut accélérer, ou au contraire ralentir les transitions vers l'emploi. Deux effets contradictoires sont en effet à l'œuvre: la formation accroît d'une part le nombre de postes susceptibles d'être proposés à ses bénéficiaires, mais peut aussi accroître les exigences salariales de ces derniers, et les pousser à refuser plus fréquemment les offres d'emploi qu'auparavant.

Le fait que la formation ait, généralement, tendance à rallonger l'épisode de chômage des individus qui en bénéficient, peut finalement s'interpréter comme une prédominance de l'effet de rétention sur les deux autres effets.

À plus long terme, la formation peut en revanche avoir pour effet d'améliorer la qualité des emplois retrouvés, et d'accroître la stabilité de ceux-ci. Les travaux ayant envisagé l'effet de la formation sur la stabilité de l'emploi retrouvé sont relativement rares. L'étude de Crépon, Ferracci, Fougère (2006), établit toutefois que la formation a pour effet d'accroître la durée du chômage, mais aussi, et dans une proportion supérieure, d'accroître la durée des emplois retrouvés. Ceci doit inciter à clarifier les objectifs assignés aux programmes de formation: accélérer le retour à l'emploi, ou accroître la stabilité de ce dernier?

Par ailleurs, les effets de la formation diffèrent selon les publics concernés. Selon LALONDE (1995), seuls quelques programmes de formation destinés aux femmes de plus de 25 ans, issues de milieux défavorisés, jouissent d'une certaine efficacité. Les programmes sont nettement moins bénéfiques pour les hommes adultes et même franchement déce-

(7) Voir en particulier Lalive, Van Ours, Zweimuller (2000) et ROSHOLM, SVARER (2004). vants pour les jeunes, surtout pour ceux de sexe masculin. Il apparaît aussi que ce sont les individus les moins qualifiés qui tirent le moins d'avantage des programmes de formation.

Ces différences dans les rendements de la formation doivent inciter à cibler les publics pour lesquels les mesures sont les plus efficaces. Ceci peut entrer en contradiction avec le principe consistant à former les individus les moins employables, qui sont aussi ceux qui accèdent le moins souvent à la formation. En conséquence, il apparaît nécessaire de clarifier l'objectif poursuivi: améliorer l'équité ou l'efficacité moyenne des dispositifs.

\section{L'incertitude concernant les rendements sociaux de la formation}

La question du rendement de la formation au-delà de la population de ses bénéficiaires est âprement débattue. La question essentielle consiste à savoir si la formation produit des externalités. Si un certain nombre d'études montrent que les externalités liées à l'éducation initiale sont significatives, il ne semble pas exister d'évaluation empirique des externalités liées à la formation professionnelle. Si ces dernières existent, il est cependant douteux qu'elles aient la même ampleur que les externalités de l'éducation initiale, laquelle agit sur l'insertion sociale en réduisant les comportements déviants, coûteux pour la collectivité (HeCKMAN, 1999). Ce type d'externalités positives concerne très peu la formation continue des adultes salariés. En revanche, de telles externalités pourraient être plus importantes pour les demandeurs d'emploi dont les périodes de chômage, surtout si elles se prolongent, sont souvent synonymes de perte de motivation et de confiance en soi. En l'absence de résultats empiriques sur ce point, il convient néanmoins de ne pas surestimer les rendements sociaux de la formation des adultes.

\section{La stratégie de l'OCDE adaptée au cas français}

\section{Mieux évaluer les dispositifs existants pour mieux cibler la formation}

L'intervention de l'État en matière de formation se justifie si les rendements sociaux de cette dernière sont supérieurs aux rendements privés. Si la formation génère des effets positifs pour la collectivité, elle peut être subventionnée. Or il est difficile, on l'a dit, d'identifier l'effet causal de la formation sur les salaires, et plus encore de mesurer les externalités de cette dernière. Sur le plan de l'évaluation, la France présente de surcroît un retard certain vis-à-vis des pays anglo-saxons et des pays nordiques. Il est donc est nécessaire d'accroître les efforts dans ce sens, afin de distinguer les publics pour lesquels la formation génère le plus fort rendement, et de permettre un ciblage plus fin. Les programmes de formation à 
destination des chômeurs ont donné lieu à un grand nombre d'évaluations sur données individuelles. Dans l'ensemble, ces travaux ne permettent pas d'établir un consensus quant à l'effet de la formation des chômeurs sur le retour à l'emploi. HeCKMAN et al. (1999) réalisent une revue de la littérature sur le sujet qui ne permet pas réellement de conclure quant à l'impact des programmes de formation sur les taux d'emploi. Selon eux, certains programmes ont des effets significatifs et positifs, tandis que d'autres montrent un impact nul, voire négatif, des mesures de formation.

Cette relative indétermination s'explique par le fait que les effets de la formation sont hétérogènes, au regard de plusieurs critères.

Les systèmes de formation diffèrent d'un pays à l'autre, et parfois d'une région à l'autre. Les processus d'affectation aux programmes sont en général variables, de même que le contenu et la qualité de ces derniers. Ceci empêche de transposer les conclusions d'une évaluation particulière à d'autres cadres institutionnels, ainsi :

-L'effet des programmes diffère selon les publics concernés. Il est donc nécessaire de réaliser des analyses stratifiées suivant certaines caractéristiques individuelles (âge, qualification, durée passée au chômage par exemple) pour obtenir des indicateurs d'impact dont le sens soit convergent.

-L'effet des programmes diffère selon les variables de résultat étudiées. L'on peut ici distinguer deux types de travaux : ceux qui s'intéressent à l'effet de la formation sur les taux d'emploi et ceux qui s'intéressent à l'effet de la formation sur les taux de transition entre le chômage et l'emploi ou, plus rarement, entre l'emploi retrouvé et le chômage.

- Du critère précédent découle une autre source d'hétérogénéité entre les travaux d'évaluation: la période sur laquelle sont suivis les individus diffère d'une étude à l'autre. Or, les effets du traitement peuvent varier dans le temps. L'OCDE (2005) note ainsi que «le profil temporel de l'impact et la nature des résultats varient suivant les programmes actifs d'emploi. Les programmes qui privilégient le retour à l'emploi ont un effet positif important à court terme, mais qui tend à décroître au fil des ans ».

Dans la mesure où les effets de la formation sont hétérogènes suivant les publics concernés, améliorer le rendement moyen des formations supposerait de concentrer les ressources sur les individus pour lesquels le rendement de la formation est le plus élevé.

\section{Procéder à des analyses coût-bénéfice de la formation}

En matière de formation des chômeurs, de nombreuses études n'évaluent l'efficacité de programmes actifs qu'à l'aune de leur effet sur la durée du chômage. L'impact des mesures sur la stabilité de l'emploi retrouvé est rarement analysé. Or il est possible que certaines mesures contribuent plutôt à augmenter la durée de l'emploi subséquent qu'à réduire la durée du chômage. Évaluant les effets des mesures de formation des chômeurs adultes en France à partir du fichier national des Assedic, CRÉPon, FerracCI et FougĖre (2006) trouvent ainsi que l'effet principal de telles mesures est d'améliorer la stabilité de l'emploi retrouvé. Toute évaluation devrait prendre en compte cet aspect, et au-delà, opérer une analyse coût-bénéfice des programmes actifs. Dans ses Perspectives de l'emploi, l'OCDE (2005) suggère d'utiliser un critère consistant à maximiser la formule $B+t W$, où $\mathrm{B}$ représente l'économie réalisée sur le versement des prestations du fait de l'accès à l'emploi, $t$ le taux d'imposition des salaires et $W$ le niveau des salaires. Cette formule mesure la valeur, pour la collectivité, des transitions du chômage vers l'emploi. Un programme efficace doit accroître cette valeur, relativement à l'absence de programme et compte tenu du coût du programme.

En France, il faut signaler que de telles analyses se heurtent à l'absence de fichier intégré retraçant l'intégralité des trajectoires individuelles et mentionnant, au cours des épisodes de chômage, le niveau de l'indemnisation, et, au cours des épisodes d'emploi, le montant des cotisations versées. Fondre en une même base de données le fichier national des Assedic et les déclarations automatisées des données sociales (DADS) permettrait de ce point de vue d'approfondir le gain pour la collectivité d'un grand nombre de mesures actives d'emploi.

\section{Accroître les incitations à investir dans la formation pour les firmes et les travailleurs: les limites du système "former ou payer" et du droit individuel à la formation}

Le système de type «former ou payer» actuellement en vigueur en France se caractérise par l'existence d'un seuil minimal de dépense, et par une absence de subvention indexée sur le volume de formation dispensée. Pour les entreprises qui dépassent ce seuil, le système n'a aucune incidence sur leurs décisions de formation, puisqu'il ne modifie pas le rendement des dépenses de formation réalisées au-delà du seuil. Dans la mesure où les rendements collectifs sont proportionnels aux rendements privés, il serait donc plus efficace de subventionner les entreprises au prorata de leurs dépenses de formation. Avec un tel système, ce sont les entreprises et les salariés qui ont le plus d'intérêts privés dans la formation continue qui sont le plus subventionnés, ce qui augmente d'autant leur volume de formation et, par conséquent, augmente aussi le plus les rendements collectifs.

Pour répondre aux inégalités d'accès à la formation, il serait ainsi possible de moduler le niveau de la subvention en fonction de la qualification, du diplôme ou de l'âge des individus formés. Ceci 
apparaît plus approprié que le système du droit individuel à la formation (DIF), établi en France en 2004, qui ne permet aux salariés de bénéficier, de manière indifférenciée, que de 20 heures de formation par an.

\section{Améliorer le fonctionnement du marché de la formation par la certification}

Dans la plupart des pays de l'OCDE, le marché de la formation professionnelle est complexe, et mal évalué (OCDE, 2006). La France ne déroge pas à cette règle. L'information sur la qualité des prestations peut toutefois être améliorée grâce à une procédure de certification. Il importe néanmoins que celle-ci soit parfaitement indépendante des opérateurs de formation, ce qui n'est, à l'heure actuelle, pas le cas en France (CAHUC, ZyLberberg, 2006).

La Stratégie pour l'emploi de l'OCDE en matière de formation est fondée sur le constat, fermement établi, qu'existent de fortes inégalités dans l'accès à la formation. Le principal reproche que l'on peut lui adresser est sans doute son excès d'optimisme quant aux bénéfices à attendre de la formation à l'échelle microéconomique. Ces bénéfices sont difficiles à évaluer, inégalement répartis dans la population, et sans doute moins importants qu'attendu. Les travaux accumulés ces dernières années enseignent ainsi que la formation, celle des chômeurs comme celle des salariés, n'est sans doute pas la «potion magique» souvent décrite.

Toutefois, il est possible que la formation professionnelle exerce des externalités positives, qui sont cependant mal mesurées à l'heure actuelle. Ceci tendrait à justifier l'intervention de l'État en matière de formation, intervention qui gagnerait toutefois à abandonner le système de "former ou payer» actuel, lequel s'avère à la fois inefficace et inéquitable, pour un système de subvention. La certification des opérateurs de formation est également une démarche essentielle.

Par-dessus tout, le manque d'évaluations empiriques empêche en France de porter un jugement global et définitif sur l'efficacité des mesures de formation. Afin d'améliorer les rendements privés et publics de la formation, il conviendrait donc de:

- clarifier les objectifs assignés à cette dernière, en particulier d'arbitrer entre les critères d'équité (former les individus les plus vulnérables, afin d'éviter le chômage de longue durée) et d'efficacité (former les individus pour lesquels les rendements sont les plus élevés);

- mieux évaluer les dispositifs existants, ce qui implique de dédier une partie des ressources engagées dans la formation aux travaux d'évaluation, et de lever les obstacles juridiques ou pratiques à la collecte de données de qualité;

- concentrer les ressources en fonction des critères préalablement établis.

\section{Bibliographie}

Brodaty T., Crépon B., Fougère D. (2007), «Les méthodes microéconométriques d'évaluation et leurs applications aux politiques actives de l'emploi», Économie et Prévision, ${ }^{\circ} 177$.

CAhUC P., Zylberberg A., (2006), «La formation professionnelle des adultes : un système à la dérive», document de travail du Conseil d'orientation pour l'emploi.

Crépon B., Ferracci M., Fougère D. (2006), “Training the Unemployed in France: Impact on Unemployment Duration and Recurrence", Miméo, Crest.

DARES (2006), «L'orientation des chômeurs vers la formation de 2002 à 2004», Premières synthèses, DARES.

Goux D., Maurin E. (2000), "Returns to Firm-Provided Training: Evidence from French Worker-Firm matched Data», Labour Economics, Vol. 7, pp. 1-19.

Heckman J. (1999), "Policies to Foster Human Capital", NBER Working Paper, No 7288.
Lalive R., Ours J. van, Zweimüller J. (2000), "The Impact of Active Labor Market Programs and Benefits Entitlement Rules On the Duration of Unemployment", IZA Discussion Paper, No 149.

Lalonde R. (1995), "The Promise of Public Sector-Sponsored Training Programs", The Journal of Economic Perspectives, Vol. 9, No 2, pp. 149-168.

Leuven E. (2004), "A Review of the Wage Returns to Private Sector Training", paper presented at the joint EC-OECD Seminar on "Human Capital and Labour and Market Performance", Bruxelles.

OCDE (2006), Perspectives de l'emploi de l'OCDE. Stimuler l'emploi et les revenus, disponible sur le site: http:// www.sourceocde.org/emploi/9264023860

Rosholm M., Svarer M. (2004), "Estimating the threat effect of Active Labour Market Programs", CAM Working Paper, 2004-14, University of Copenhaguen. 